\title{
Investigation and Synthesis of Some Novel Spiro Heterocycles Related to Indoline Moiety
}

\author{
Y. A. El-Ossaily", R. M. Zaki, and S. A. Metwally \\ Department of Chemistry, Faculty of Science, Assiut University, Assiut 71516, Egypt
}

Received 8 January 2014, accepted in revised form 20 March 2014

\begin{abstract}
Reactions of indole-2,3-dione 1 with 2-mercaptobenzimidazole, o-phenylenediamine, 2aminophenol, 2-aminobenzothiazole, 2-aminobenzimidazole and 3-methyl-1-phenyl-2pyrazolin-5-one were carried out to give compounds spiroindolethiazetobenzimidazole $\mathbf{2}$, spirobenzimidazole(oxazole)indoline 3a,b, benzothiazol(imidazol) iminoindolinone $\mathbf{4 a , b}$ and methyloxoindolylidenepyrazolone $\mathbf{5}$ respectively. Compound $\mathbf{5}$ was reacted with 2 aminophenol as well as $o$-phenylenediamine to give new spirooxazepine and diazepine derivatives $\mathbf{6 a}, \mathbf{b}$ respectively. Reaction of $\mathbf{5}$ with nitrogen nucleophiles as well as carbon nucleophiles was investigated to furnish new spiro heterocycles 7-11. The reaction of 2-(2oxo-1,2-dihydroindol-3-ylidene)malononitrile compound 12 with 3-methyl-1-phenyl-2pyrazoline-5-one was carried out to give spiroindolopyranopyrazolo derivative 13 . Compounds $\mathbf{4 a , b}$ was reacted with thioglycolic acid to give thiazolidinone derivatives 14a,b. Epoxidation of 5 using monoperoxyphthalic acid magnesium salt hexahydrate and hydrogen peroxide were executed to afford the novel dispiro (2-pyrazolin oxiraneindoline)dione compound $\mathbf{1 5}$. The chemical structures of the synthesized compounds were well established by elemental and spectral analyses.
\end{abstract}

Keywords: Spiroheterocycles; Epoxidation; Diazapines, Oxazepines; Spirothiazolidinone.

(C) 2014 JSR Publications. ISSN: 2070-0237 (Print); 2070-0245 (Online). All rights reserved. doi: http://dx.doi.org/10.3329/jsr.v6i2.17590 J. Sci. Res. 6 (2), 293-307 (2014)

\section{Introduction}

Among the various heterocyclic systems, indole holds a prominent place because it is present as a core unit in a number of compounds possessing a broad spectrum of biological activates $[1,2]$. It is well known that the spiro-oxindole heterocyclic framework is an important structural motif in biologically relevant compounds as natural products and pharmaceuticals, e.g., surugatoxin, horsfiline, spirotryprostatin A and B, elacomine, gelsemine, alstonisine and strychnofoline [3-9].

\footnotetext{
* Corresponding author: yasserabdelmoez@yahoo.com
} 
Further, the chemistry of spiro-indoles in which an indole ring is joined to sulfur and nitrogen containing heterocycles at the C-3 position through a spiro carbon atom is of great interest due to their physiological and biological activities [10-14]. Spiro (indolethiazolidinones) are known to possess various biological activities including antiinflammatory [15], antimicrobial [16], bacteriostatic [17], anticonvulsant [18] and used as antifungal agents [19]. Pyridopyrimidine and their derivatives are of high interest in organic chemistry due to their potential biological and pharmacological activities such as antiviral [20-21], anti-inflammatory [22], insecticidal [23], antifolate [24], tyrosine kinase inhibitor [25], antimicrobial [26], calcium channel antagonists [27], antileishmanial [28], diuretic and potassium-sparing [29]. On the other hand the structure activity relationship (SAR) of benzodiazepines, benzoxazepines had been studied [30-34]. Diazepines have strong central depressant, anticonvulsant and anxiolytic activity [35-39]. It is of interest to note that pyrazoles are reported as well-known pharmaceuticals [40-43]. From this point of view and in continuation of our previous work [44-47], our aim is to design some new spiro heterocycles containing indoline moiety with expected high biological activity. Further in this paper we present the full experimental details of some novel spiroheterocycles [48-54].

\section{Experimental}

The time required for completion of each reaction was monitored by TLC. Melting point was measured by (Gallen-Kamp) apparatus and was uncorrected. Elemental analysis was performed with elemental analysen systeme GmbH. Verio EI. IR spectra were recorded with Shimadzu 470 Infrared Spectrophotometer ( $\mathrm{KBr}$ wafer technique). ${ }^{1} \mathrm{H}$ and ${ }^{13} \mathrm{C}$ NMR spectra were taken with a NMR LA 400 (Joel) (400 and $100 \mathrm{MHz}$ respectively) with TMS as internal standard. Mass spectrometric analysis was recorded with Joel-JMS 600.

\subsection{Spiro (indole-3,2`1, 3-thiazeto [3, 2-a]) benzimidazole-2-one (2)}

A mixture of indole-2,3-dione $1(0.002 \mathrm{~mol})$ and 2-mercaptobenzimidazole $(0.002 \mathrm{~mol})$ in absolute ethanol $(20 \mathrm{~mL})$ in the presence of triethylamine $(1 \mathrm{~mL})$ was heated under reflux for $6 \mathrm{~h}$. The reaction mixture was cooled to room temperature, the solvent was evaporated under reduced pressure and the resultant residue was recrystallized from ethanol as orange crystals; yield (80\%), m.p.:190-192 ${ }^{\circ} \mathrm{C}, \mathrm{IR}(\mathrm{KBr}): v_{\max }\left(\mathrm{cm}^{-1}\right): 3250(\mathrm{NH}), 3050(\mathrm{CH}$ aromatic), $1720(\mathrm{C}=\mathrm{O}), 1620(\mathrm{C}=\mathrm{N}) ;{ }^{1} \mathrm{H}-\mathrm{NMR}\left(\mathrm{DMSO}_{6} \mathrm{~d}_{6}\right): \delta$ 6.61-7.70 (m, 8H, Ar-H), $10.35(\mathrm{~s}, 1 \mathrm{H}, \mathrm{NH})$. EI-MS: $\mathrm{m} / \mathrm{z}(\%)=279\left(\mathrm{M}^{+}, 91.9\right) .{ }^{13} \mathrm{C}-\mathrm{NMR}\left(\mathrm{DMSO}-\mathrm{d}_{6}\right): 75.62(\mathrm{C} 8$ spirothiazetoindole), 115.24-129.85 (ArH), 130.50 (C9), 135.64 (C1), 142.22 (C10), 161.87(C7), 166.35 (CO indole). Anal. Calcd. For: $\mathrm{C}_{15} \mathrm{H}_{9} \mathrm{~N}_{3} \mathrm{OS}$ (279.322): C, 64.50, H, 3.25, N, 15.04, S, 11.48. Found: C, 64.43, H, 3.22, N, 14.97, S, 11.52. 


\subsection{Synthesis of compounds $3 a, b$}

A mixture of 1 (0.002 mol), o-phenylenediamine and/or $o$-aminophenol $(0.002 \mathrm{~mol})$ and triethylamine $(1 \mathrm{~mL})$ was refluxed in absolute ethanol $(20 \mathrm{~mL})$ for $6 \mathrm{~h}$. The reaction mixture was cooled to room temperature and the resultant solid was filtered, and recrystallized from a mixture of ethanol: water $(2: 1)$.

\subsubsection{Spiro (2, 3-dihydrobenzimidazole-2, 3'-indoline)-2'-one (3a)}

Obtained by reaction of isatin and $o$-phenylenediamine as yellow crystals in $80 \%$ yield; m.p.: $170-172^{\circ} \mathrm{C}, \operatorname{IR}(\mathrm{KBr}): v_{\max }\left(\mathrm{cm}^{-1}\right): 3260(\mathrm{NH}), 3050(\mathrm{CH}$ arom $), 1710(\mathrm{C}=\mathrm{O}) ;{ }^{1} \mathrm{H}-$ NMR (DMSO-d $\left.{ }_{6}\right): \delta 6.00-7.75(\mathrm{~m}, 8 \mathrm{H}, \mathrm{ArH}), 9.60(\mathrm{~s}, 2 \mathrm{H}, 2 \mathrm{NH}$ benzimidazole), $10.30(\mathrm{~s}$, $1 \mathrm{H}$, NH indole). Anal. Calcd. For: $\mathrm{C}_{14} \mathrm{H}_{11} \mathrm{~N}_{3} \mathrm{O}$ (237.26): C, 70.87; H, 4.67; N, 17.71. Found: C, 71.05; H, 4.62; N, 17.73.

\subsubsection{Spiro [benzoxazole-2, $3{ }^{`}$-indoline]-2'-one (3b)}

Obtained by reaction of isatin and $o$-aminophenol as orange crystals in $60 \%$ yield; m.p. 208-210 ${ }^{\circ} \mathrm{C}$; IR(KBr): $v_{\max }\left(\mathrm{cm}^{-1}\right): 3250(\mathrm{NH}), 3050\left(\mathrm{CH}\right.$ aromatic), $1710(\mathrm{C}=\mathrm{O}) ;{ }^{1} \mathrm{H}-\mathrm{NMR}$ (DMSO-d ${ }_{6}$ ): $\delta$ 6.90-7.75 (m, 8H, ArH), 9.70 (s, 1H, NH benzothiazole), 10.35 (s, 1H, NH indole). EI-MS: $\mathrm{m} / \mathrm{z}(\%)=238\left(\mathrm{M}^{+}, 100\right)$. Anal.Calcd. For: $\mathrm{C}_{14} \mathrm{H}_{10} \mathrm{~N}_{2} \mathrm{O}_{2}$ (238.24): Anal. Calcd. For: C, 70.58; H, 4.23; N, 11.75. Found: C, 70.43; H, 4.25; N, 11.78.

\subsection{Synthesis of compounds $4 a, b$}

A mixture of 1 (0.002 mol), 2-aminobenzothiazole and/or 2-aminobenzimidazole (0.002 mol) and triethylamine $(1 \mathrm{~mL})$ was refluxed in absolute ethanol $(20 \mathrm{~mL})$ for $6 \mathrm{~h}$. The reaction mixture was evaporated under reduced pressure. The resultant solid was recrystallized from ethanol.

\subsubsection{3-(1H-benzo[d]thiazol-2-ylimino)-1, 3-dihydroindolin-2-one (4a)}

Red crystals; yield (72\%); m.p.:218-220 ${ }^{\circ} \mathrm{C}$. IR(KBr): $v_{\max }\left(\mathrm{cm}^{-1}\right): 3200(\mathrm{NH}), 3050(\mathrm{CH}$ aromatic), $1710(\mathrm{C}=\mathrm{O}) ; 1620(\mathrm{C}=\mathrm{N}) ;{ }^{1} \mathrm{H}-\mathrm{NMR}\left(\mathrm{DMSO}_{6}\right)$ : $\delta$ 6.61-7.70 (m, 8H, Ar-H), 10.35 (s, 1H, NH indole). Anal. Calcd. For $\mathrm{C}_{15} \mathrm{H}_{9} \mathrm{~N}_{3} \mathrm{OS}$ (279.32): C, 64.50; H, 3.24; N, 15.04, S, 11.47. Found: C, 64.44; H, 3.44; N, 15.22, S, 11.66.

\subsubsection{3-(1H-benzo[d]imidazol-2-ylimino)-1, 3-dihydroindolin-2-one (4b)}

Orange crystals; yield (65\%); m.p.: 188-190 ${ }^{\circ} \mathrm{C} . \mathrm{IR}(\mathrm{KBr}): v_{\max }\left(\mathrm{cm}^{-1}\right): 3250(\mathrm{NH}), 3050$ $\left(\mathrm{CH}\right.$ aromatic), $1700(\mathrm{C}=\mathrm{O}), 1620(\mathrm{C}=\mathrm{N}) ;{ }^{1} \mathrm{H}-\mathrm{NMR}\left(\mathrm{DMSO}^{-} \mathrm{d}_{6}\right): \delta$ 6.61-7.70 (m, 8H, Ar- 
H), 9.80 (s, 1H, NH benzimidazole), 10.35 (s, 1H, NH indole). Anal. Calcd. For $\mathrm{C}_{15}$ $\mathrm{H}_{10} \mathrm{~N}_{4} \mathrm{O}$ (262.27): C, 68.69; H, 3.84; N, 21.36. Found: C, 68.71; H, 3.96; N, 21.15.

\subsection{3-Methyl-4-(2'-oxoindole-3-ylidene)-1-phenyl-pyrazol-5-one (5)}

A mixture of $1(0.002 \mathrm{mmol})$ and 3-methyl-1-phenyl-2-pyrazoline-5-one in absolute ethanol $(20 \mathrm{~mL})$ and TEA $(1 \mathrm{~mL})$ was heated under reflux for $6 \mathrm{~h}$. The reaction mixture was cooled to room temperature and the resultant solid was collected by filtration, dried and recrystallized from ethanol as brown crystals; yield $(90 \%)$, m.p. $158-160^{\circ} \mathrm{C}$; $\operatorname{IR}(\mathrm{KBr})$ : $v_{\max }\left(\mathrm{cm}^{-1}\right): 3150(\mathrm{NH}), 3050(\mathrm{CH}$ aromatic), 1720 (CO indole), $1680(\mathrm{C}=\mathrm{O}$ pyrazole), $1620(\mathrm{C}=\mathrm{N}), 1600(\mathrm{C}=\mathrm{C}) ;{ }^{1} \mathrm{H}-\mathrm{NMR}\left(\mathrm{DMSO}_{6}\right): \delta 2.30\left(\mathrm{~s}, 3 \mathrm{H}, \mathrm{CH}_{3}\right), 6.90-7.75(\mathrm{~m}, 9 \mathrm{H}$, Ar-H), 10.35 (s, 1H, NH indole). EI-MS: m/z $(\%)=302\left(\mathrm{M}^{+}-1,46\right)$. Anal. Calcd. For: $\mathrm{C}_{18} \mathrm{H}_{13} \mathrm{~N}_{3} \mathrm{O}_{2}$ (303.32): C, 71.27; H, 4.31; H, 13.85. Found: C, 71.20; H, 4.25; N, 13.96.

\subsection{Synthesis of compounds $6 a, b$}

A mixture of chalcone $5(0.01 \mathrm{~mol}), o$-aminophenol and/or $o$-phenylenediamine $(0.01$ $\mathrm{mol})$ and few drops of piperdine was refluxed in ethanol $(50 \mathrm{~mL})$ for $4 \mathrm{~h}$, then glacial acetic acid $(10 \mathrm{~mL})$ was added to the reaction mixture then heating was continued for further $2 \mathrm{~h}$. The reaction mixture was cooled to room temperature, left overnight and the resultant solid was filtered, dried and recrystallized from ethanol.

2.5.1. 3`-Methyl-2-oxo-1`-phenyl-1,2,10`-trihydro-1`H-spiroindole-3,4`-pyrazolo[4,5-c] benzo[b] oxazepine (6a)

Grey crystals, yield (68\%); m.p. 136-138 ${ }^{\circ} \mathrm{C}$; IR(KBr): $v_{\max }\left(\mathrm{cm}^{-1}\right): 3150(\mathrm{NH}), 3050(\mathrm{CH}$ aromatic), $1710(\mathrm{C}=\mathrm{O}), 1620(\mathrm{C}=\mathrm{N}), 1605\left(\mathrm{C}=\mathrm{C}\right.$ str). ${ }^{1} \mathrm{H}-\mathrm{NMR}\left(\mathrm{DMSO}-\mathrm{d}_{6}\right): \delta 2.30(\mathrm{~s}, 3 \mathrm{H}$, $\mathrm{CH}_{3}$ ), 6.75-7.89 (m, 13H, Ar-H), 9.70 (s, 1H, NH oxazepine), 10.35 (s, 1H, NH indole).

${ }^{13} \mathrm{C}-\mathrm{NMR}$ (DMSO-d $\left.{ }_{6}\right): 15.70\left(\mathrm{CH}_{3}\right.$ pyrazole), $74.20(\mathrm{C} 4), 116.05-129.80(\mathrm{ArH}), 135.95$ (C5), 139.50 (C19 aromatic), 140.15 (C6), 142 (C13), 144.80 (C2), 145.50 (C11), 162.40 (C3), $167.60(\mathrm{CO})$. EI-MS: $\mathrm{m} / \mathrm{z}(\%)=394\left(\mathrm{M}^{+}, 24\right)$. Anal. Calcd. For: $\mathrm{C}_{24} \mathrm{H}_{18} \mathrm{~N}_{4} \mathrm{O}_{2}$ (394.43): C, 73.08; H, 4.59; N, 14.20. Found: C, 73.12; H, 4.58; H, 14.35.

\subsubsection{3`-Methyl-2-oxo-1`-phenyl-1,2,10`-trihydro-1 ${ }^{\prime} H$-spiroindole-3,4`-pyrazolo[4,5-c]} benzo $[b]$ diazepine $(6 b)$

Brown crystals, yield (75\%); m.p.:226-228 ${ }^{\circ}$; IR(KBr): $v_{\max }\left(\mathrm{cm}^{-1}\right): 3150(\mathrm{NH}), 3050(\mathrm{CH}$ aromatic), $1720(\mathrm{C}=\mathrm{O}), 1620(\mathrm{C}=\mathrm{N}), 1595\left(\mathrm{C}=\mathrm{C}\right.$ str). ${ }^{1} \mathrm{H}-\mathrm{NMR}\left(\mathrm{DMSO}_{\mathrm{d}}\right)$ ): $2.35(\mathrm{~s}, 3 \mathrm{H}$, $\mathrm{CH}_{3}$ ), 6.70- 7.75 (m, 13H, Ar-H), 9.60 (s, 2H, 2NH diazepine), 10.20 (s, 1H, NH indole). Anal. Calcd. For: $\mathrm{C}_{24} \mathrm{H}_{19} \mathrm{~N}_{5} \mathrm{O}_{2}$ (409.44), C, 70.40; H, 4.67; N, 17.10. Found: C, 70.52; H, $4.88 ; \mathrm{N}, 17.20$. 


\subsection{Synthesis of spiropyrazolo [5,4-d] pyrimidine derivatives $7 a, b$.}

A mixture of chalcone $5(0.01 \mathrm{~mol})$, urea and/or thiourea $(0.01 \mathrm{~mole})$ was refluxed in ethanol $(50 \mathrm{~mL})$ for $6 \mathrm{~h}$. Then the product was precipitated, collected by filtration, dried, and recrystallized from ethanol.

2.6.1. 5`Methyl-2-oxo-7`-phenyl-1,2,1`,2`,3`-pentahydro-7`H-spiroindole-3,4`-pyrazolo [5,4-d] pyrimidine-2`one (7a)

Black crystals, yield (82\%); m.p.: 240-242 ${ }^{\circ} \mathrm{C}$; IR(KBr): $v_{\max }\left(\mathrm{cm}^{-1}\right): 3150(\mathrm{NH}), 3030($ $\mathrm{CH}$ aromatic), $1720(\mathrm{C}=\mathrm{O}$ indole $), 1680(\mathrm{C}=\mathrm{O}$ pyrimidine $), 1600(\mathrm{C}=\mathrm{C}$ str $) .{ }^{1} \mathrm{H}-\mathrm{NMR}$ $\left(\mathrm{DMSO}_{-} \mathrm{d}_{6}\right): \delta 2.30\left(\mathrm{~s}, 3 \mathrm{H}, \mathrm{CH}_{3}\right), 6.90-7.81(\mathrm{~m}, 9 \mathrm{H}, \mathrm{Ar}-\mathrm{H}), 9.70,9.85(2 \mathrm{~s}, 2 \mathrm{H}, 2 \mathrm{NH}$ pyrimidine), 10.30 (s, 1H, NH indole); Anal. Calcd. For: $\mathrm{C}_{19} \mathrm{H}_{15} \mathrm{~N}_{5} \mathrm{O}_{2}$ (345.36) C, 66.07; H, 4.37; N, 20.27. Found: C, 66.22; H, 4.52; N, 20.12.

2.6.2. 5`Methyl-2-oxo-7`-phenyl-1,2,1 ‘2`,3`-pentahydro-7`H-spiroindole-3,4`-pyrazolo [5,4-d]pyrimidine-2`-thione (7b)

Black crystals, yield (80\%); m.p.: 182-184 ${ }^{\circ} \mathrm{C}$; IR(KBr): $v_{\max }\left(\mathrm{cm}^{-1}\right): 3150(\mathrm{NH}), 3030(\mathrm{CH}$ aromatic), $1710(\mathrm{C}=\mathrm{O}), 1600\left(\mathrm{C}=\mathrm{C}\right.$ str). ${ }^{1} \mathrm{H}-\mathrm{NMR}\left(\mathrm{DMSO}_{6}\right): \delta 2.30\left(\mathrm{~s}, 3 \mathrm{H}, \mathrm{CH}_{3}\right), 6.61-$ $7.70(\mathrm{~m}, 9 \mathrm{H}, \mathrm{Ar}-\mathrm{H}), 9.60,9.80(2 \mathrm{~s}, 2 \mathrm{H}, 2 \mathrm{NH}$ pyrimidine), 10.35 (s, 1H, NH indole). Anal.Calcd. For: $\mathrm{C}_{19} \mathrm{H}_{15} \mathrm{~N}_{5} \mathrm{OS}$ (367.42), C, 63.14; H, 4.18; N, 19.37; S, 8.87. Found: C, $63.15 ; \mathrm{H}, 4.22 ; \mathrm{N}, 19.55 ; \mathrm{S}, 8.90$.

\subsection{Synthesis of spiropyrazolo [3,4-c] pyrazole derivatives 8 a.e}

The chalcone $5(0.001 \mathrm{~mol})$ and an excess of the appropriate hydrazine $(1 \mathrm{mmol})$ was refluxed for $2 \mathrm{~h}$ in ethanol $(20 \mathrm{~mL})$. The reaction mixture was cooled to room temperature and the solvent was removed under reduced pressure. The residual product recrystallized from ethanol.

\subsubsection{3`-Methyl-2-oxo-1`-phenyl-1,2,5`6`-tetrahydro-1 `H-spiroindole-3,4`-pyrazolo} [3,4-c] pyrazol-2-one (8a)

Obtained as black crystals by the reaction of chalcone 5 and hydrazine hydrate in $88 \%$ yield; m.p.: $108-110^{\circ} \mathrm{C}$; $\mathrm{IR}(\mathrm{KBr}) v_{\max }\left(\mathrm{cm}^{-1}\right): 3150(\mathrm{NH}), 3050(\mathrm{CH}$ aromatic $), 1710$ $(\mathrm{C}=\mathrm{O}), 1620(\mathrm{C}=\mathrm{N}), 1595\left(\mathrm{C}=\mathrm{C}\right.$ str). ${ }^{1} \mathrm{H}-\mathrm{NMR}\left(\mathrm{DMSO}_{-} \mathrm{d}_{6}\right): \delta 2.30\left(\mathrm{~s}, 3 \mathrm{H}, \mathrm{CH}_{3}\right), 6.61-$ $7.70(\mathrm{~m}, 9 \mathrm{H}, \mathrm{Ar}-\mathrm{H}), 9.70,9.85(2 \mathrm{~s}, 2 \mathrm{H}, 2 \mathrm{NH}$ pyrazole), 10.35 (s, 1H, NH indole). Anal.Calcd. For: $\mathrm{C}_{18} \mathrm{H}_{15} \mathrm{~N}_{5} \mathrm{O}$ (317.35), C, 68.12; H, 4.76; N, 22.06. Found: C, 68.30; H, $4.88 ; \mathrm{N}, 22.23$. 
2.7.3. 3`-Methyl-2-oxo-1`,6`-diphenyl-1,2,5`6`-tetrahydro-1`H-spiroindole-3,4`pyrazolo [3,4-c]pyrazol-2-one (8b)

Obtained by the reaction with phenylhydrazine as yellow crystals in $90 \%$ yield; m.p.:164$166^{\circ} \mathrm{C}$; IR(KBr): $v_{\max }\left(\mathrm{cm}^{-1}\right): 3150(\mathrm{NH}), 3050(\mathrm{CH}$ aromatic $), 1710(\mathrm{C}=\mathrm{O}), 1620(\mathrm{C}=\mathrm{N})$, $1600\left(\mathrm{C}=\mathrm{C}\right.$ str). ${ }^{1} \mathrm{H}-\mathrm{NMR}\left(\mathrm{DMSO}_{6}\right) \delta: 2.30\left(\mathrm{~s}, 3 \mathrm{H}, \mathrm{CH}_{3}\right), 6.65-7.70(\mathrm{~m}, 14 \mathrm{H}, \mathrm{ArH})$, $9.65\left(\mathrm{~s}, 1 \mathrm{H}, \mathrm{NH}\right.$ pyrazole), $10.35\left(\mathrm{~s}, 1 \mathrm{H}, \mathrm{NH}\right.$ indole). Anal.Calcd. For: $\mathrm{C}_{24} \mathrm{H}_{19} \mathrm{~N}_{5} \mathrm{O}$ (393.45), C, 73.26; H, 4.86; N, 22.06. Found: C, 73.20; H, 4.80; N, 22.16.

2.7.3. 6`-(m-Bromophenyl)-3`-methyl-2-oxo-1`-phenyl-1,2,5`6`-tetrahydro-1`Hspiroindole- 3,4`-pyrazolo [3,4-c]pyrazol-2-one (8c)

Obtained by the reaction with $m$-bromophenylhydrazine as yellow crystals in $82 \%$ yield, m.p.: 216-218 $\mathrm{C}$ dec.; IR(KBr): $v_{\max }\left(\mathrm{cm}^{-1}\right): 3200(\mathrm{NH}), 3050(\mathrm{CH}$ aromatic $), 1720(\mathrm{C}=\mathrm{O})$ , $1620(\mathrm{C}=\mathrm{N}), 1600\left(\mathrm{C}=\mathrm{C}\right.$ str). ${ }^{1} \mathrm{H}-\mathrm{NMR}\left(\mathrm{DMSO}-\mathrm{d}_{6}\right) \delta: 3.00\left(\mathrm{~s}, 3 \mathrm{H}, \mathrm{CH}_{3}\right), 6.60-7.51(\mathrm{~m}$, $13 \mathrm{H}, \mathrm{ArH}), 9.90(\mathrm{~s}, 1 \mathrm{H}, \mathrm{NH}$ pyrazole), 10.35 (s, 1H, NH indole). Anal, Calcd. For: $\mathrm{C}_{24} \mathrm{H}_{18} \mathrm{BrN}_{5} \mathrm{O}$ (471.48), C, 61.14; H, 3.84; Br, 16.76; N, 14.85. Found: C, 61.22; H, 3.82; $\mathrm{Br}, 16.70 ; \mathrm{N}, 14.89$.

2.7.4. 6`-(m-Nitrophenyl)-3`-methyl-2-oxo-1`-phenyl-1,2,5`6`-tetrahydro-1`Hspiroindole-3,4-pyrazolo[3,4-c]pyrazol-2-one (8d)

Obtained by the reaction with $m$-nitrophenylhydrazine as brown crystals in(60\%)yield; m.p.:215-217 ${ }^{\circ} \mathrm{C}$; IR(KBr): $v_{\max }\left(\mathrm{cm}^{-1}\right): 3250(\mathrm{NH}), 3050(\mathrm{CH}$ aromatic $), 1720(\mathrm{C}=\mathrm{O})$, $1620(\mathrm{C}=\mathrm{N}), 1600\left(\mathrm{C}=\mathrm{C}\right.$ str). ${ }^{1} \mathrm{H}-\mathrm{NMR}\left(\mathrm{DMSO}_{\mathrm{d}}\right) \delta: 2.30\left(\mathrm{~s}, 3 \mathrm{H}, \mathrm{CH}_{3}\right), 6.80-7.80(\mathrm{~m}$, $13 \mathrm{H}, \mathrm{ArH}), 9.80$ (s, $1 \mathrm{H}, \mathrm{NH}$ pyrazole), 10.35 (s, 2H, 2NH). Anal.Calcd. For: $\mathrm{C}_{24} \mathrm{H}_{18} \mathrm{~N}_{6} \mathrm{O}_{3}$ (438.44), C, 65.74; H, 4.13; N, 19.16. Found: C, 65.88; H, 4.20, N, 19.20.

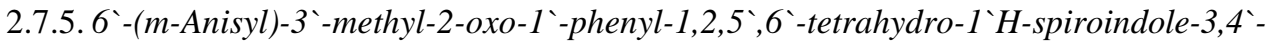
pyrazolo [3,4-c]pyrazol-2-one (8e)

Obtained by the reaction with $m$-methoxyphenylhydrazine as pale yellow crystals in (88\%) yield; m.p.:174-176 ${ }^{\circ} \mathrm{C}$; $\mathrm{IR}(\mathrm{KBr}) v_{\max }\left(\mathrm{cm}^{-1}\right): 3200(\mathrm{NH}), 3050(\mathrm{CH}$ aromatic) , $1715(\mathrm{C}=\mathrm{O}), 1620(\mathrm{C}=\mathrm{N}), 1595(\mathrm{C}=\mathrm{C}$ str $) .{ }^{1} \mathrm{H}-\mathrm{NMR}\left(\mathrm{DMSO}_{6}\right) \delta: 2.50\left(\mathrm{~s}, 3 \mathrm{H}, \mathrm{CH}_{3}\right), 3.2$ $\left(\mathrm{s}, 3 \mathrm{H}, \mathrm{OCH}_{3}\right), 6.90-7.95(\mathrm{~m}, 13 \mathrm{H}, \mathrm{ArH}), 11.61(\mathrm{~s}, 2 \mathrm{H}, 2 \mathrm{NH})$. Anal. Calcd. For: $\mathrm{C}_{25} \mathrm{H}_{21} \mathrm{~N}_{5} \mathrm{O}_{2}$ (423.47), C, 70.980; H, 4.99; N, 16.53. Found: C, 70.88; H, 4.80; N, 16.70.

\subsection{Synthesis of spiroindolopyrano[2,3-c]pyrazole derivatives}

A mixture of chalcone $5(0.01 \mathrm{~mol})$ and the appropriate active methylene compound $(0.01$ $\mathrm{mol})$ in absolute ethanol $(20 \mathrm{~mL})$ in the presence of triethylamine $(1 \mathrm{~mL})$ was refluxed for 
6h. The precipitated product was collected by filtration, dried, and recrystallized from ethanol.

\subsubsection{5`-Acetyl-3`,6`dimethyl-2-oxo-1`-phenyl-1,2-dihyro-1`H-spiroindole-3,4`-pyrano} [2,3-c] pyrazole (9)

Obtained as black crystals by the reaction with acetyl acetone in 68\% yield; m.p. 252$254^{\circ} \mathrm{C}$; IR(KBr): $v_{\max }\left(\mathrm{cm}^{-1}\right): 3360(\mathrm{NH}), 3050(\mathrm{CH}$ aromatic $), 1710\left(\mathrm{CO}, \mathrm{COCH}_{3}\right)$, $1650\left(\mathrm{C}=\mathrm{O}\right.$ amidic), $1620(\mathrm{C}=\mathrm{N}), 1590\left(\mathrm{C}=\mathrm{C}\right.$ str). ${ }^{1} \mathrm{H}-\mathrm{NMR}\left(\mathrm{DMSO}_{6}\right) \delta: 2.50\left(_{\mathrm{s}}, 3 \mathrm{H}\right.$, $\left.\mathrm{CH}_{3}\right), 2.30$ (s, 3H, $\left.\mathrm{CH}_{3}\right), 2.70\left(\mathrm{~s}, 3 \mathrm{H}, \mathrm{CH}_{3}\right), 6.60-7.90$ (m, 9H, Ar-H), 11.35 (s, 1H, NH). Anal. Calcd. For: $\mathrm{C}_{25} \mathrm{H}_{21} \mathrm{~N}_{5} \mathrm{O}_{2}$ (385.42), C, 71.67; H, 4.96; H, 10.90. Found: C, 71.53; H, 5.02; H, 10.88 .

2.8.2. Ethyl-6 -amino-3`-methyl-2-oxo-1,2-dihydro-1 ${ }^{\prime} H$-spiroindole-3,4`-pyrano[2,3-c] pyrazole-5'-carboxylate (10)

Obtained by the reaction with ethylcyanoacetate as brown crystals in (92\%) yield; m.p.: 208-210 ${ }^{\circ} \mathrm{C}, \mathrm{IR}(\mathrm{KBr}): v_{\max }\left(\mathrm{cm}^{-1}\right): 3350,3300\left(\mathrm{NH}_{2}\right), 3200(\mathrm{NH}), 3050(\mathrm{CH}$ aromatic) , 1720 ( CO ester) ,1700 (C=O indole ), $1620(\mathrm{C}=\mathrm{N}), 1600(\mathrm{C}=\mathrm{C}$ str $) .{ }^{1} \mathrm{H}-\mathrm{NMR}\left(\mathrm{DMSO}-\mathrm{d}_{6}\right)$ $\delta: 1.10-1.30\left(\mathrm{t}, J=7.5 \mathrm{~Hz}, 3 \mathrm{H}, \mathrm{CH}_{3}\right), 2.30\left(\mathrm{~s}, 3 \mathrm{H}, \mathrm{CH}_{3}\right), 4.10-4.30(\mathrm{q}, J=6.0 \mathrm{~Hz}, 2 \mathrm{H}$, $\left.\mathrm{OCH}_{2}\right), 4.75\left(\mathrm{~s}, 2 \mathrm{H}, \mathrm{NH}_{2}\right), 6.80-7.60(\mathrm{~m}, 9 \mathrm{H}, \mathrm{ArH}), 9.45(\mathrm{~s}, 1 \mathrm{H}, \mathrm{NH})$. EI-MS: $m / z(\%)=$ $416\left(\mathrm{M}^{+}\right.$, 14.6). Anal. Calcd. For: $\mathrm{C}_{23} \mathrm{H}_{20} \mathrm{~N}_{4} \mathrm{O}_{4}$ (416.43), C, 66.33; H, 4.84; N, 13.45. Found: C, 66.23; H, 5.02; N, 13.30.

2.8.3. 3-Dicyanomethyl-3-(3`-methyl-5`oxo-1`-phenyl- 2-pyrazolin-4-yl)indole-2-one (11)

Obtained as dark brown crystals by the reaction with malononitrile in $90 \%$ yield, m.p.: 228-230 ${ }^{\circ} \mathrm{C}$ dec.; IR(KBr): $v_{\text {max }}\left(\mathrm{cm}^{-1}\right): 3300(\mathrm{NH}), 3050(\mathrm{CH}$ aromatic), $2200(\mathrm{CN}), 1710$ ( CO indole), $1680\left(\mathrm{C}=\mathrm{O}\right.$ pyrazole), $1620(\mathrm{C}=\mathrm{N}) .{ }^{1} \mathrm{H}-\mathrm{NMR}\left(\mathrm{DMSO}_{6}\right) \delta: 1.30(\mathrm{~s}, 1 \mathrm{H}$, $\mathrm{CH}), 1.60$ (s, 1H, CH), 2.30 (s, 3H, $\left.\mathrm{CH}_{3}\right), 6.80-7.60$ (m, 9H, ArH), 9.45 (s, 1H, NH). EIMS: $\mathrm{m} / \mathrm{z}(\%)=369\left(\mathrm{M}^{+}, 1.40\right)$. Anal. Calcd. For: $\mathrm{C}_{21} \mathrm{H}_{15} \mathrm{~N}_{5} \mathrm{O}_{2}$ (369.38), C, 68.28; H, 4.09; N, 18.95. Found: C, 68.12; H, 4.23; N, 19.02 .

2.8.4. 6`-Amino-5`-cyano-3`-methyl-1`-phenyl-1,2-dihydro-1`H-spiroindole-3,4`-pyrano [2,3-c] pyrazole (13)

A mixture of 2-(2-oxo-1,2-dihydroindol-3-ylidene)malononitrile (12) (0.01 mol) and 3methyl-1-phenyl-2 pyrazoline-5-one $(0.01 \mathrm{~mol})$ in absolute ethanol $(20 \mathrm{~mL})$ in the presence of triethylamine $(1 \mathrm{~mL})$ was refluxed for $3 \mathrm{~h}$. The precipitated product was collected by filtration, dried, and recrystallized from ethanol as colorless crystals, yield (95\%); m.p.: 254-256 ${ }^{\circ} \mathrm{C}$; IR(KBr): $v_{\max }\left(\mathrm{cm}^{-1}\right): 3350,32300\left(\mathrm{NH}_{2}\right), 3200(\mathrm{NH}), 3050(\mathrm{CH}$ aromatic), $2200(\mathrm{CN}), 1700(\mathrm{C}=\mathrm{O}), 1620(\mathrm{C}=\mathrm{N}), 1600\left(\mathrm{C}=\mathrm{C}\right.$ str); ${ }^{1} \mathrm{H}-\mathrm{NMR}\left(\mathrm{DMSO}-\mathrm{d}_{6}\right)$ $\delta: 2.50\left(\mathrm{~s}, 3 \mathrm{H}, \mathrm{CH}_{3}\right), 4.75\left(\mathrm{~s}, 2 \mathrm{H}, \mathrm{NH}_{2}\right), 6.55-7.75(\mathrm{~m}, 9 \mathrm{H}, \mathrm{ArH}), 9.45(\mathrm{~s}, 1 \mathrm{H}, \mathrm{NH}) .{ }^{13} \mathrm{C}-$ 
NMR (DMSO-d $\left.)_{6}\right) 15.46\left(\mathrm{CH}_{3}\right.$ pyrazole), $55.60(\mathrm{C} 4), 65.80(\mathrm{C} 5), 115.20(\mathrm{CN}), 119.60-$ 129.46 (ArH), 135.28 (C14), 145.62 (C3), 164.24 (C6), 168.40 (CO). EI-MS: $m / z(\%)=$ $369\left(\mathrm{M}^{+}\right.$, 100). Anal. Calcd. For: $\mathrm{C}_{21} \mathrm{H}_{15} \mathrm{~N}_{5} \mathrm{O}_{2}$ (369.38), C, 68, 28; H, 4.09; N, 18.95 . Found: C, 68.12; H, 4.23; N, 19.02.

\subsection{Synthesis of spirothiazolidineindoline 14a,b}

A mixture of $\mathbf{4 a}$ or $\mathbf{4 b}(0.01 \mathrm{~mol})$, thioglycolic acid $(0.01 \mathrm{~mL})$ and $30 \mathrm{~mL}$ THF was heated under reflux for $4 \mathrm{~h}$ in presence of anhydrous $\mathrm{ZnCl}_{2}$. The reaction mixture was cooled and filtered. The filtrate was evaporated under reduced pressure. The residual solid product was recrystallized from ethanol.

2.9.1. 3'-(1,3-benzothiazol-2-yl)-4H'-spiro[indole-3,2'-[1, 3]thiazolidine-2,4'(1H)-dione (14a)

Brown plates; yield 60\%, m.p.: 258-260 ${ }^{\circ} \mathrm{C}(\mathrm{dec}$.$\left.) ; IR( \mathrm{KBr}\right) v\left(\mathrm{~cm}^{-1}\right): 3200(\mathrm{NH}), 3050(\mathrm{CH}$ aromatic), 1720(CO indole), $1680\left(\mathrm{C}=\mathrm{O}\right.$ thiazole), $1600\left(\mathrm{C}=\mathrm{C}\right.$ str); ${ }^{1} \mathrm{H}-\mathrm{NMR}$ (DMSO$\left.\mathrm{d}_{6}\right) \delta: 4.30\left(\mathrm{~s}, 2 \mathrm{H}, \mathrm{CH}_{2}\right), 6.65-7.80(\mathrm{~m}, 8 \mathrm{H}, \mathrm{ArH}), 10.35$ (s, 1H, NH). Anal. Calcd. For: $\mathrm{C}_{17} \mathrm{H}_{11} \mathrm{~N}_{3} \mathrm{O}_{2} \mathrm{~S}_{2}$ (353.42), C, 57.77; H, 3.13; N, 11.88; S, 18.14. Found: C, 57.66; H, 3.12, $\mathrm{N}, 11.92 ; \mathrm{S}, 18.20$.

2.9.2. 3'-(1,3-benzimidazole-2-yl)-4H'-spiro[indole-3,2'-[1,3]thiazolidine-2,4'(1H)-dione (14b)

Black Crystals; yield 65\%, m.p.: 218-220 $\mathrm{C}$, IR(KBr): $v_{\text {max }}\left(\mathrm{cm}^{-1}\right): 3300(\mathrm{NH}), 3050(\mathrm{CH}$ aromatic), 1720 (CO indole), $1680\left(\mathrm{C}=\mathrm{O}\right.$ thiazole). ${ }^{1} \mathrm{H}-\mathrm{NMR}\left(\mathrm{DMSO}-\mathrm{d}_{6}\right) \delta: 4.30$ (s, $2 \mathrm{H}$, $\left.\mathrm{CH}_{2}\right), 6.60-7.75(\mathrm{~m}, 8 \mathrm{H}, \mathrm{ArH}), 10.30(\mathrm{~s}, 1 \mathrm{H}, \mathrm{NH}$ indole $), 11.35(\mathrm{~s}, 1 \mathrm{H}, \mathrm{NH}$ benzimidazole). Anal.Calcd. For: $\mathrm{C}_{17} \mathrm{H}_{12} \mathrm{~N}_{4} \mathrm{O}_{2} \mathrm{~S}$ (336.37), C, 60.70; H, 3.59; N, 16.65; S, 9.53. Found: C, 60.65, H, 3.72; N, 16.45; S, 9.60.

\subsection{3-Methyl-1-phenyldispiro (2-pyrazolin-4, 3`oxirane-2`3“-indoline)-5,2"-dione (15)}

Method A: The chalcone 5 (0.004 mol) was suspended in acetonitrile $(25 \mathrm{~mL})$, and aqueous hydrogen peroxide solution $(30 \%, 10 \mathrm{~mL})$ was added to the stirred suspension followed by aqueous sodium hydroxide solution $(10 \%, 1-3 \mathrm{~mL})$, As soon as the color of the original chalcone disappeared, the reaction mixture was filtered. The resulting filtrate was diluted with water and neutralized with oxalic acid $(5 \%)$ to give a yellow precipitate which was collected by filtration. The resulting solid precipitate was recrystallized from ethanol in $40 \%$ yield and the m.p. was $220-222^{\circ} \mathrm{C}$.

Method B: A solution of $\mathrm{Mg}$ monoperoxy phthalic acid hexahydrate (4 eq.) and the chalcone 5 in absolute methanol $(4 \mathrm{~mL} / \mathrm{mmol})$ was stirred for $4 \mathrm{~h}$. The resulting mixture was filtered and concentrated. The residue was triturated with water to give a yellow precipitate which was collected, dried and recrystallized from ethanol in yield $81 \%$ and 
m.p. was $220-222^{\circ} \mathrm{C}$; IR(KBr): $v_{\max }\left(\mathrm{cm}^{-1}\right): 3300(\mathrm{NH}), 3050(\mathrm{CH}$ aromatic), $1720(\mathrm{C}=\mathrm{O}$ indole), $1680\left(\mathrm{C}=\mathrm{O}\right.$ pyrazole), $1620(\mathrm{C}=\mathrm{N}), 1110(\mathrm{C}-\mathrm{O}), 900$ (epoxide ring); ${ }^{1} \mathrm{H}-\mathrm{NMR}$ $\left(\mathrm{DMSO}_{-} \mathrm{d}_{6}\right) \delta: 2.50$ (s, 3H, $\left.\mathrm{CH}_{3}\right), 6.65-7.75(\mathrm{~m}, 9 \mathrm{H}, \mathrm{ArH}), 10.35$ (s, 1H, NH). EI-MS: m/z $(\%)=319\left(\mathrm{M}^{+}\right.$, 56). Anal. Calcd. For: $\mathrm{C}_{18} \mathrm{H}_{13} \mathrm{~N}_{3} \mathrm{O}_{2}$ (319.31) $\mathrm{C}, 67.70 ; \mathrm{H}, 4.10 ; \mathrm{N}, 13.15$. Found: C, 67.72; H, 4.05; N, 13.22.

\section{Results and Discussions}

Refluxing of isatin with 2-mercaptobenzimidazole in ethanol and in presence of triethylamine as a basic catalyst furnished the new spiroindolethiazetobenzimidazole derivative $\mathbf{2}$, The chemical structure of thiazeto derivative $\mathbf{2}$ was established by elemental and spectral analyses. IR spectrum showed absorption band at $1720 \mathrm{~cm}^{-1}$ characteristic for one carbonyl group of isatin and at $3250 \mathrm{~cm}^{-1}$ for NH group. ${ }^{1} \mathrm{H}-\mathrm{NMR}$ showed multiplet signals centered at 6.61-7.70 characteristic for aromatic protons. Mass spectrum showed a molecular ion peak at $\mathrm{m} / \mathrm{z} 279$ with high intensity. Also, isatin was refluxed with $o$ phenylenediamine as well as with 2-aminophenol in ethanol and in presence of TEA to give spirobenzimidazoleindoline $\mathbf{3 a}$ and benzoxazoleindoline $\mathbf{3 b}$ derivatives respectively. The structures of the latter compounds were elucidated using IR, ${ }^{1} \mathrm{H}-\mathrm{NMR}$ and mass spectrometric analyses which were in full agreement with the suggested structures. Mass spectrum showed a molecular ion peak at 238 as a base peak. Condensation of compound 1 with 2-aminobenzothiazole or 2-aminobenzimidazole was carried out in ethanol and triethylamine as a basic catalyst to give the corresponding Schiff's bases $\mathbf{4 a}$ and $\mathbf{4 b}$ respectively. Isatin reacted with 3-methyl-1-phenyl-2-pyrazoline-5-one in ethanol and triethylamine to give the corresponding chalcone 5.The chemical structure of chalcone $\mathbf{5}$ was confirmed by TLC, IR, ${ }^{1} \mathrm{H}-\mathrm{NMR}$ and mass spectra. IR spectrum showed two absorptions at $1720 \mathrm{~cm}^{-1}$, characteristic for carbonyl group of indole moiety, $1680 \mathrm{~cm}^{-1}$ characteristic for carbonyl group of pyrazolone moiety. ${ }^{1} \mathrm{H}-\mathrm{NMR}$ spectrum showed singlet signals at $\delta 2.30$ and $10.35 \mathrm{ppm}$ for $\mathrm{CH}_{3}$ group of pyrazole, $\mathrm{NH}$ group of isatin respectively (Scheme 1). Compound 5 was subjected to extensive study through the reaction with nitrogen nucleophiles. Reaction of compound 5 with 2-aminophenol, $o_{\text {- }}$ phenylenediamine, gave the new spiro indole pyrazolo benzoxazepine 6a and spiro indole pyrazolo benzodiazepine $\mathbf{6 b}$ respectively.

The chemical structure of compound $\mathbf{6 a}$ was elucidated on the basis of elemental and spectral analyses. IR spectrum of $\mathbf{6 a}$ showed absorption bands at $3150 \mathrm{~cm}^{-1}$ for $\mathrm{NH}$ and at 1710 and $1605 \mathrm{~cm}^{-1}$ for $\mathrm{CO}$ group of indole and $\mathrm{C}=\mathrm{C}$ of pyrazole respectively. ${ }^{1} \mathrm{H}-\mathrm{NMR}$ spectrum showed singlet signal at $\delta 2.30$ for $\mathrm{CH}_{3}$ of pyrazole moiety and singlet at 9.60, 10.35 characteristic for $\mathrm{NH}$ groups of oxazepine and indole respectively. Mass spectrum of $6 \mathrm{a}$ showed a molecular ion peak at 394. While reaction of chalcone 5 with urea, thiourea, hydrazine, phenylhydrazine, $m$-bromophenyl hydrazine, $m$-nitrophenyl hydrazine and $p$-methoxy phenylhydrazine in ethanol afforded spiro indole pyrazolopyrimidines 7a,b and spiropyrazolopyrazoles 8a-e respectively (Scheme 2). It was found that the percentage of yield of products 8a-e depended on the nucleophilicity of substituent on the pyrazole ring. Where, the weak nucleophilic group decreases the percentage of yield according to its intensity as in $\mathbf{8 c}$ and $\mathbf{8 d}$ while the strong nucleophilic 
group increases the percentage of yield. But in case of $p$-methyl phenylhydrazine as strong nucleophile which contain electron releasing $\left(\mathrm{OCH}_{3}\right)$ group, the yield doesn't increase (may be) due to steric interaction of $\mathrm{OCH}_{3}$ group with the spiroindolopyrazole nucleus.

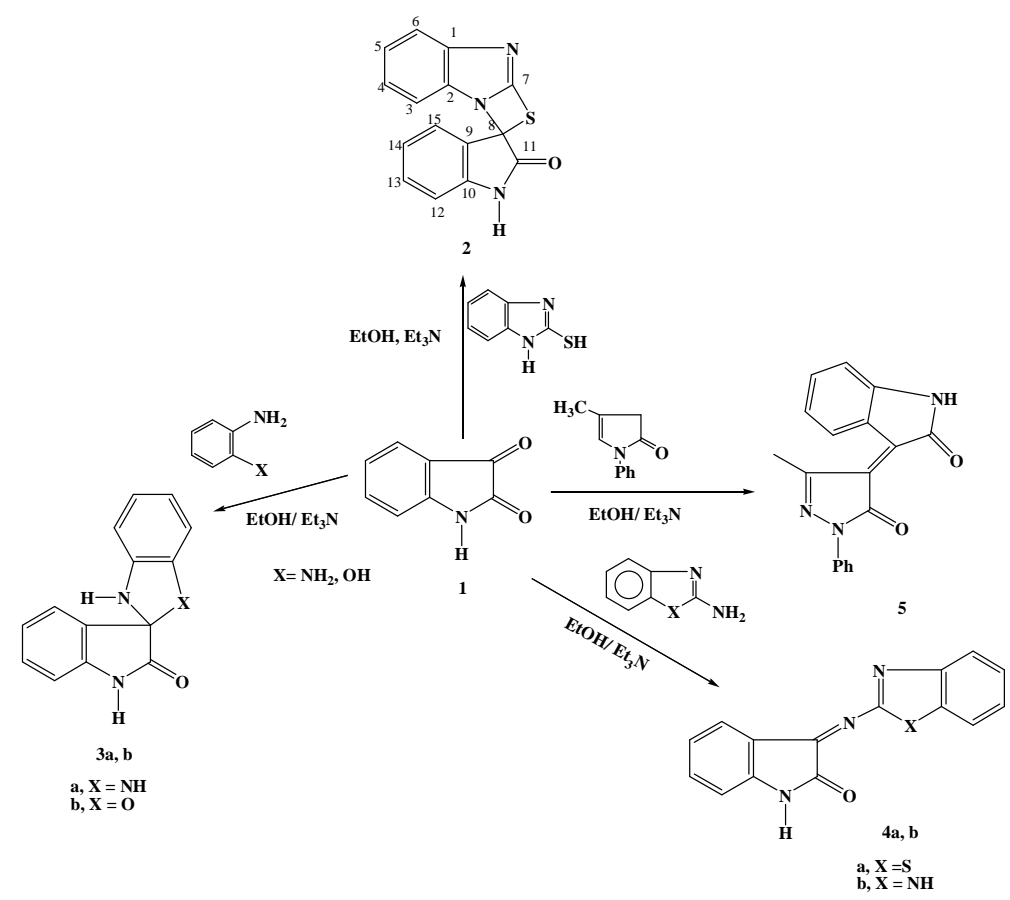

Scheme 1. Synthesis of spiroindolethiazetobenzimidazole 2, spirobenzimidazole(oxazole)indoline 3a, b benzothiazol(imidazol)iminoindolinone 4a,b and methyloxoindolylidene1 pyrazolone 5

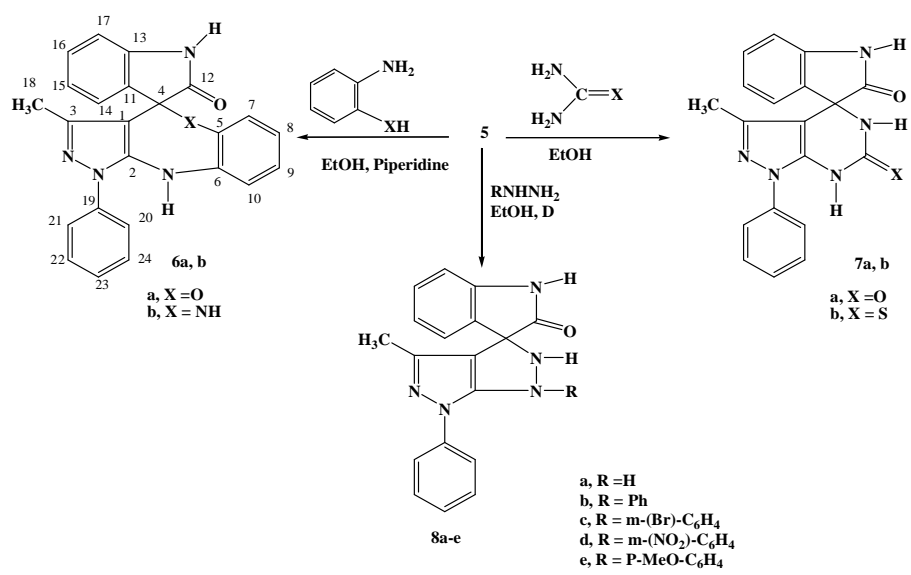

Scheme 2. Synthesis of spirooxazepine, diazepine, spiropyrazolo[5,4-d]pyrimidine and spiropyrazolo[3,4-c]pyrazole. 
The suggested mechanism for the formation of compounds $\mathbf{6 a}, \mathbf{b}$ was described as 1, 2Michael addition of nucleophilic $\mathrm{OH}$ or $\mathrm{NH}_{2}$ group in o-aminophenol or o-phenylene diamine to the $\mathrm{C}=\mathrm{C}$ bond of $\alpha, \beta$-unsaturated carbonyl of pyrazolone followed by cyclization through nucleophilic addition of $\mathrm{NH}_{2}$ to the carbonyl group of pyrazolone and elimination of water molecule to form the oxazepine (diazepine) compounds $\mathbf{6 a}, \mathbf{b}$ as shown in the following scheme.

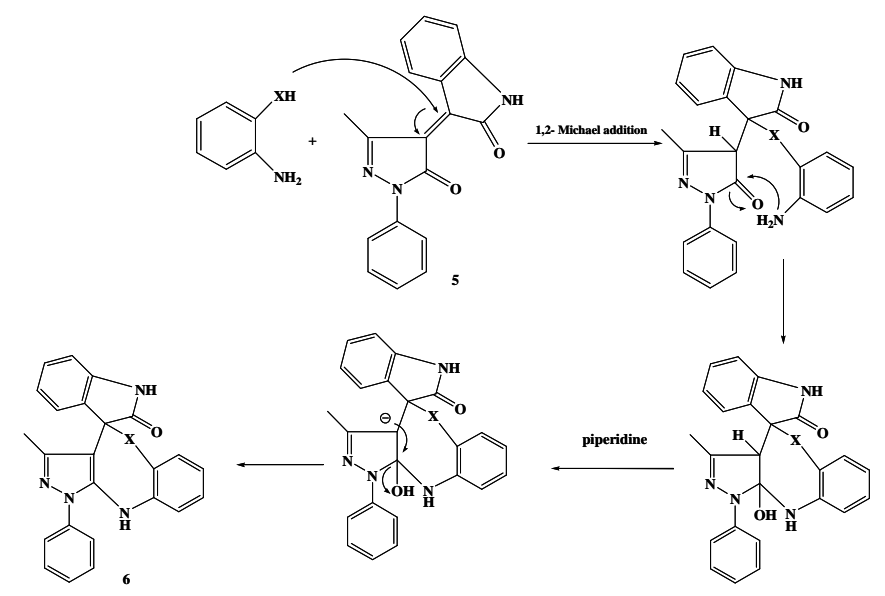

Scheme 3. The suggested reaction mechanism for formation of spirooxazepine $6 \mathrm{a}$ and diazepine $6 \mathrm{~b}$.

On the other hand, 3-methyl-4-(2`-oxoindole-3-ylidene)-1-phenyl-pyrazol-5-one compound (5) reacted with acetylacetone as well as ethyl cyanoacetate as active methylene derivatives in ethanol and in presence of triethylamine to give the new acetylspiroindolepyranopyrazole $\mathbf{9}$ and amino spiroindolepyranopyrazole acetic acid ethyl ester $\mathbf{1 0}$ in quantitative yield. Compound $\mathbf{5}$ was treated with malononitrile in ethanol and in presence of triethylamine to give 3-indolone derivative $\mathbf{1 1}$ not the aminospiroindolepyranopyrazole carbonitrile derivative $\mathbf{1 3}$ which was clearly shown by IR spectroscopy. Further investigation was done through treatment of isatin with malononitrile to give the 3-dicyanomethylene derivative 12 which reacted with 3-methyl1-phenyl-2-pyrazoline-5-one in ethanol and triethylamine to furnish the cyclic spiro derivative $\mathbf{1 3}$ in quantitative yield (Scheme 4). The structure of compound $\mathbf{1 3}$ was established by spectral analysis. IR spectrum showed absorption bands at 3350, 3300 and $3200 \mathrm{~cm}^{-1}$ for $\mathrm{NH}_{2}, \mathrm{NH}$ groups and absorption band at $2200 \mathrm{~cm}^{-1}$ for $\mathrm{CN}$ group. ${ }^{1} \mathrm{H}-\mathrm{NMR}$ showed singlet signal at $\delta 4.75$ for $\mathrm{NH}_{2}$ group and singlet signal at $\delta 9.45$ for $\mathrm{NH}$ group. Mass spectrum of compound 13 showed a molecular ion peak at 369 as a base peak.

Alternative route to synthesize spiro-indoline thiazolidinones 14a,b was carried out through the reaction of Schiff's bases $\mathbf{4 a , b}$ with thioglycolic acid (Scheme 5). IR spectrum of compound 14a showed two absorption bands at $1720 \mathrm{~cm}^{-1}$ characteristic for carbonyl group of indole moiety and $1680 \mathrm{~cm}^{-1}$ characteristic for carbonyl group of thiazolidine 
moiety. ${ }^{1} \mathrm{H}-\mathrm{NMR}$ showed two singlet signals at $\delta 4.30$ for $\mathrm{CH}_{2}$ group of thiazolidine ring and at $\delta 10.35$ for $\mathrm{NH}$ group.

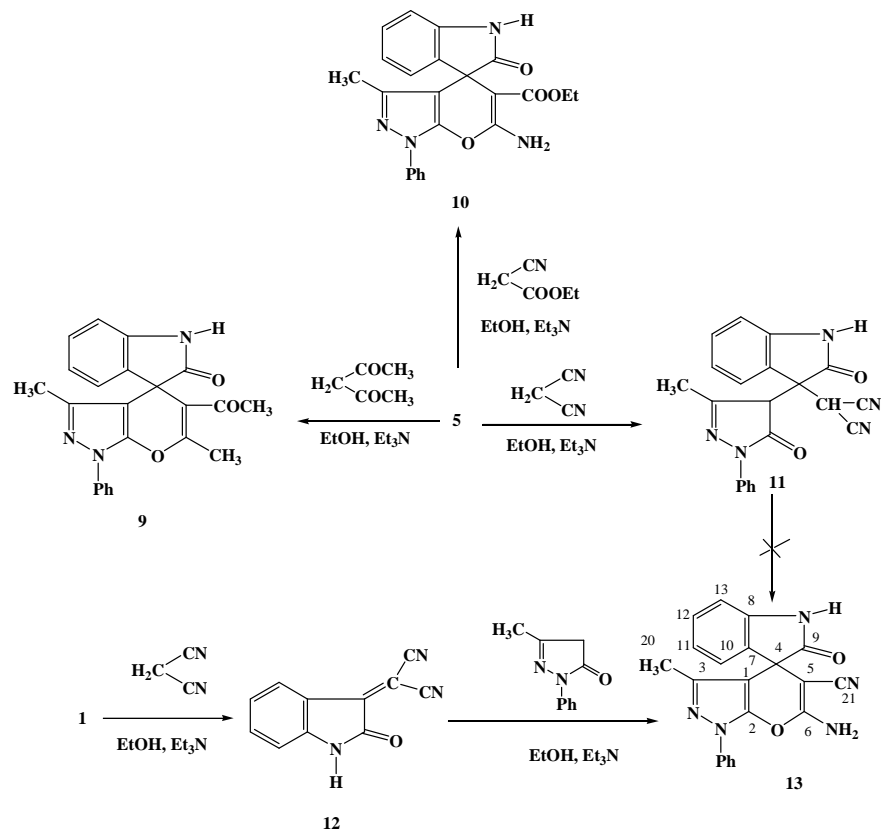

Scheme 4. Synthesis of spiroindolopyranopyrazole derivatives.

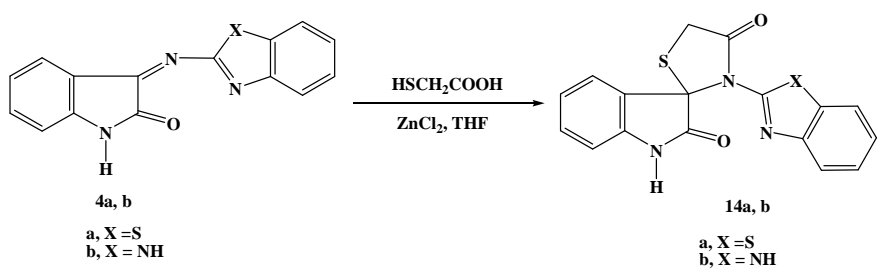

Scheme 5. Synthesis of spirothiazolidineindoline derivatives.

Epoxidation of compound $\mathbf{5}$ with hydrogen peroxide as well as monoperoxy phthalic acid magnesium salt hexahydrate gives the new epoxide derivative 15. Compound $\mathbf{1 5}$ which was synthesized by two routes are identical in all aspects (Scheme 6). The chemical structure of compound $\mathbf{1 5}$ was proved by elemental and spectral data. IR spectrum showed absorption bands at $1720 \mathrm{~cm}^{-1}$ characteristic for carbonyl group of indole moiety, 1680 $\mathrm{cm}^{-1}$ characteristic for pyrazole moiety and absorption band at 1110 for $\mathrm{C}-\mathrm{O}$ of epoxide ring. ${ }^{1} \mathrm{H}-\mathrm{NMR}$ spectrum showed multiplet signals at 6.65-7.75 for aromatic protons and singlet signal at 10.35 for NH group. Mass spectrum showed a molecular ion peak at 319 . 


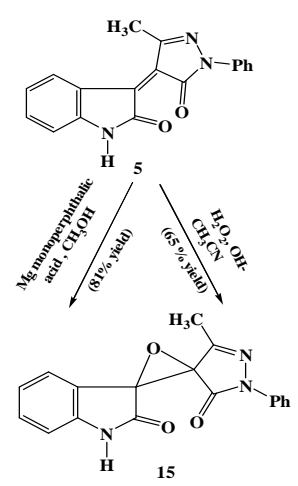

Scheme 6. Synthesis of 3- Methyl-1-phenyldispiro (2-pyrazolin-4, 3`-oxirane-2`, 3“-indoline)-5,2"dione

\section{Conclusion}

The reactions discussed in this paper describe a simple facile synthetic procedure to prepare new spiroheterocyclic derivatives of diazapines, oxazepines and Spirothiazolidinone, which might have important biological applications.

\section{References}

1. M. Wolf and A. A. Mascitti, U.S. Patent 3395, 156, (1968); Chem. Abstr. 69, 96504 (1968).

2. G. Winters and N. D. Mola, Ger. Patent 2442, 667, 1975; Chem. Abstr. 83, 28096 (1975).

3. R. M. Williams and R. J. Cox, Acc. Chem. Res. 36, 127 (2003). http://dx.doi.org/10.1021/ar020229e

4. D. J. F. M. Silva, S. J. Garden, and A. C. Pinto, J. Braz. Chem. Soc. 12, 273 (2001). http://dx.doi.org/10.1590/S0103-50532001000300002

5. M. Y. Chang, C. L. Pai, and Y. H. Kung, Tetrahedron Lett. 46, 8463 (2005). http://dx.doi.org/10.1016/j.tetlet.2005.10.015

6. S. P. Baran and R. M. Richter, J. Am. Chem. Soc. 127, 15394 (2005). http://dx.doi.org/10.1021/ja056171r

7. S. T. Hilton, T. C. T. Ho, G. Pijevaljcic, and K. hones, Org. Lett. 2, 2639 (2000). http://dx.doi.org/10.1021/o10061642

8. T. Kosuge, K. Tsuji, K. Hirai, K. Yamaguchi, T. Okamoto, and Y. Iitaka, Tetrahedron Lett. 22, 3417 (1981). http://dx.doi.org/10.1016/S0040-4039(01)81920-1

9. M. J. Kornet and A. P. Thio, J. Med. Chem. 19, 892 (1976). http://dx.doi.org/10.1021/jm00229a007

10. K. Stratmann, R. E. Moore, R. Bonjouklia, J. B. Deeter, G. M. L. Patterson, S. Shaffer, C.D. Smith, and T. A. Smitka, J. Am. Chem. Soc. 116, 9935 (1994). http://dx.doi.org/10.1021/ja00101a015

11. J. W. Skiles and D. Menil, Tetrahedron Lett. 31, 7277 (1990). http://dx.doi.org/10.1016/S0040-4039(00)88543-3

12. H. B. Yang, Y. Wei, and M. Shi, Tetraherdron 69, 4088 (2013). http://dx.doi.org/10.1016/j.tet.2013.03.062

13. M. S. Shmidt, I. A. Perillo, M. Gonzalez, and M. M. Blanco, Tetrahedron Lett. 53, 2514 (2012). http://dx.doi.org/10.1016/j.tetlet.2012.03.010 
14. J. Quiroga, S. Portllo, A. Perez, J. Galvez, R. Abonia, and B. Insuasty, Tetrahedron Lett. 52, 2664 (2011). http://dx.doi.org/10.1016/j.tetlet.2011.03.067

15. A. Verma and S. K. Saraf, Eur. J. Med. Chem. 13, 879 (2008).

16. E. Piscopo, M. V. Diurno, O. Mazzoni, and A. M. Ciaccio, Boll. Soc. Ital. Biol. Sper. 66, 1181 (1990).

17. V. Sehgal, P. Singh, A. Dandia, and R. Bohra, Acta Cryst. C50, 1156 (1994). http://dx.doi.org/10.1107/S0108270193012697

18. J. S. Birdar and S. Y. Manjunath, Indian J. Chem. 43B, 389 (2004).

19. A. Dandia, R. Singh, S. Khaturia, C. Merienne, G. Morgant, and A. Loupy, Bioorg. Med. Chem. 14, 2409 (2006). http://dx.doi.org/10.1016/j.bmc.2005.11.025

20. G. Singh, G. Singh, A. K. Yadav, and A. K. Mishra, Indian J. Chem. 41B, 430 (2002).

21. N. Kumar, G. Singh, and A. K. Yuadav, Heteroatom Chem. 12, 52 (2001). http://dx.doi.org/10.1002/1098-1071(2001)12:1<52::AID-HC11>3.0.CO;2-0

22. N. Ghilsoo,; M. Y. Cheol,; K. Euikyung, K. R. Chung, H. K. Joong,; H. S. Jung, and H. K. Sung, Bioorg. Med. Chem. Lett. 11, 611 (2001).

23. R. E. Heckler and G. P. Jourdan, Eur. Pat. Appl. 414, 386 (1991); Chem. Abstr. 115, 71630 (1991).

24. A. Rosowsky, C. E. Mota, and S. F. Queener, J. Heterocyclic Chem. 32, 335 (1995).

25. A. M. Thompson, A. J. Bridges, D. W. Fry, A. J. Kraker, and W. A. Denny, J. Med. Chem. 38, 3780 (1995). http://dx.doi.org/10.1021/jm00019a007

26. I. O. Donkor, C. L. Klein, L. Liang, N. Zhu, E. Bradley, and A. M. Clark, J. Pharm. Sci. 84, 661 (1995). http://dx.doi.org/10.1002/jps.2600840526

27. A. Pastor, R. Alajarin, J. J. Vaquero, B. J. Alvarez, J. F. Gasa, C. M. Sunkel, J. G. Priego, I. Fonseca, and A. J. Sanz, Tetrahedron 50, 8085 (1994). http://dx.doi.org/10.1016/S00404020(01)85291-1

28. N. K. Satti, K. A. Suri, O. P. Sun, and A. Kapil, Indian J. Chem. 32B, 978 (1993).

29. A. Monge, M. V. Martinez, C. Sammartin, F. J. Fernandez, M. C. Ochoa, C. Berllver, P. Artigas, and A. E. Fernandez, Eur. J. Med. Chem. 24, 209 (1989). http://dx.doi.org/10.1016/0223-5234(89)90001-9

30. L. H. Sternack, J. Med. Chem. 1, 22 (1979).

31. Childress in Burger's Medicinal Chemistry, S. J. ed. M. E. Wolff, $4^{\text {th }}$ edition (John Wiley, New York, 1981), Part III, 990.

32. Y. D. Kulkarni, S. H. R. Abdi, and V. L. Sharma, J. Ind. Chem. Soc. 63, 425 (1986).

33. Z. Vejdelek, Z. Polivka, and M. Protiva, Collect. Czechosl. Chem. Comm. 50, 1064 (1985). http://dx.doi.org/10.1135/cccc19851064

34. R. A. Mane and D. B. Ingle, Ind. J. Chem. 21B, 973 (1982).

35. M. Babbini, M. Gaiardi, and M. Bartolett, Life Sci. 25, 159 (1979). http://dx.doi.org/10.1016/0024-3205(79)90484-3

36. C. Braestrup, R. Albrechtsen, and R. F. Squires, Nature (London) 269, 702 (1977). http://dx.doi.org/10.1038/269702a0

37. S. E. File and S. Pellow, Psychopharmacology 80, 166 (1983). http://dx.doi.org/10.1007/BF00427962

38. B. A. Weissman, J. Cott, S. M. Paul, and P. Skolnick, Eur. J. Pharmacol 90, 1449 (1983). http://dx.doi.org/10.1016/0014-2999(83)90229-7

39. S. E. File, A. R. Green, D. J. Nutt, and N. D. Vincent, Psychopharmacol. 82, 199 (1984). http://dx.doi.org/10.1007/BF00427773

40. F. T. Coppe and M. M. Fawzi, J. Heterocycl. Chem. 34, 1693 (1977). http://dx.doi.org/10.1002/jhet.5570340608

41. A. Steinmetz and L. F. Tietze, Ger Offen. DE 19, 627, 002 (CL CO7D231/22) 8 Jan. 1998, Appl. 19, 627, 002, 5 Jul 1996, 14; Chem Abstr. 128, 114946e (1998).

42. Y. Suzuki, Y. Takemura, K. Iwamoto, T. Higashino, and A. Miyashito, Chem. Pharm. Bull. 46, 199 (1998). http://dx.doi.org/10.1248/cpb.46.199 
43. S. S. Bhagwat, C. Lee, M. D. Cowart, J. Mckie, and A. L. Grillot, PCT Int. Appl. WO 9846, 605 (CL. CO7D471/04) 22 Oct. 1998, US Appl. 818, 216, 16 Apr. 1997, 172 pp; Chem. Abstr. 129, $316240 b$ (1996).

44. S. A. M. Metwally, T. A. Mohamed, O. S. Moustafa, and Y. A. El-Ossaily, Chem. Heterocyclic Comp. 11, 1659 (2010).

43. M. F. El-Zohry, Y. A. El-ossaily, T. A. Mohamed, and E. M. Hussein, Phosphorus, Sulfur, Silicon \& Relat. Elem. 183, 2095 (2008). http://dx.doi.org/10.1080/10426500701849287

44. M. F. El-Zohry, Y. A. El-Ossaily, T. A. Mohamed, E. M. Hussein, Heterocycles 75 (4), 955 (2008). http://dx.doi.org/10.3987/COM-07-11277

45. M. F. El-Zohry, Y. A. El-Ossaily, T. A. Mohamed, and E. M. Hussein, Heterocyclic Commun. 14 (3), 195 (2008). http://dx.doi.org/10.3987/COM-07-11277

46. A. A. Abdel-Hafez and M. F. El-Zohry, Heterocyclic Commun. 7 (6), 583 (2001). http://dx.doi.org/10.1515/HC.2001.7.6.583

47. M. S. Mohamed, S.M. Awad, and N.M. Ahmed, J. Appl. Pharmaceut. Sci. 1 (5), 76 (2011).

48. M. N. Ibrahim, M. F. El-Messmary, and M. G. Aelarfi, E-Journal Chem. 7 (1), 55 (2010). http://dx.doi.org/10.1155/2010/604549

49. E. M. Hussein and M. I. Abdel-Monem, Arkivok 10, 85 (2011). http://dx.doi.org/10.3998/ark.5550190.0012.a07

50. A. D. Dandia, G. Sharma, R. Singh, and A. Laxkar, Arkivoc 14, 100 (2009).

51. H. M. Mamun, M. A. Foysal, M. Mahabub, and Al- Amin, J. Sci. Res. 2 (2), 322 (2010). DOI: $10.3329 /$ jsr.v2i2.3731

52. A. Dandia, R. Singh, S. Khaturia, C. Merienne, G. Morgant, and A. Loupy, Bioorganic \& Medicinal Chem. 14, 2409 (2006). http://dx.doi.org/10.1016/j.bmc.2005.11.025 